\title{
Danish Childhood Cancer Registry
}

This article was published in the following Dove Press journal:

Clinical Epidemiology

25 October 2016

Number of times this article has been viewed

\author{
Henrik Schrøder' \\ Catherine Rechnitzer ${ }^{2}$ \\ Peder Skov Wehner ${ }^{3}$ \\ Steen Rosthøj ${ }^{4}$ \\ Jens Kjølseth Møller ${ }^{5}$ \\ Birgitte Lausen ${ }^{2}$ \\ Gitte Petersen ${ }^{2}$ \\ Mette Nørgaard ${ }^{6}$ \\ 'Department of Pediatrics, Aarhus \\ University Hospital, Aarhus, \\ ${ }^{2}$ Department of Pediatrics and \\ Adolescent Medicine, Rigshospitalet, \\ Copenhagen University Hospital, \\ Copenhagen, ${ }^{3}$ Department of \\ Pediatric Hematology and Oncology, \\ Hans Christian Andersen Children's \\ Hospital, Odense University Hospital, \\ Odense, ${ }^{4}$ Department of Pediatrics, \\ Aalborg University Hospital, Aalborg, \\ ${ }^{5}$ Department of Clinical Microbiology, \\ Vejle Sygehus, Vejle, ${ }^{6}$ Department \\ of Clinical Epidemiology, Aarhus \\ University Hospital, Aarhus, Denmark
}

Correspondence: Henrik Schrøder Department of Pediatrics, Aarhus University Hospital, DK 8200 Aarhus, Denmark

Tel $+457845 \quad 1422$

Fax +45 7845 I710

Email henrik.schroeder@skejby.rm.dk
Aim of database: The overall aim is to monitor the quality of childhood cancer care in Denmark; to register late effects of treatment; to analyze complications of permanent central venous catheters (CVCs); to study blood stream infections in children with cancer; and to study acute toxicity of high-dose methotrexate infusions in children with leukemia.

Study population: All children below 15 years of age at diagnosis living in Denmark diagnosed after January 1, 1985 according to the International Classification of Diseases 10, including diagnoses DC00-DD48.

Main variables: Cancer type, extent of disease, treatment, participation in international studies, recurrence of malignant disease, survival, yearly follow-up status, causes of death, and development of secondary malignancies. Type of CVC, causes for removal of the CVC, type of blood stream infection, pathogens isolated, antimicrobial sensitivity, and outcome of antimicrobial chemotherapy.

Descriptive data: Since 1985, 4,944 children below 15 years of age have been registered in the database. There has been no significant change in the incidence of childhood cancer in Denmark since 1985. The 5-year survival has increased significantly since 1985 and is now $86 \%$. The median number of days from diagnosis to initiation of therapy is 7 days and in $80 \%$ of the children less than 14 days. Clinical data of $95 \%$ of the patients are reported to open international studies.

Conclusion: The survival of Danish children with cancer since 2003 compares favorably with other international population-based studies. The annual reports support the collaboration within pediatric oncology in Denmark.

Keywords: childhood cancer, epidemiology, survival, complications

\section{Aim of the database}

The Danish Childhood Cancer Registry (DCCR) is a nationwide clinical quality database whose aim it is to report on the quality indicators outlined in Table 1 in all Danish children below 15 years of age at the time of cancer diagnosis. Overall survival and relapse-free survival are analyzed with respect to cancer type, extent of disease, treatment modalities, response to treatment, and recurrence of malignant disease. Since 2014 DCCR also serves as the basis for a national quality study of the use of central venous catheters (CVCs) and of the occurrence of blood stream infections in children with cancer. The aim of this part of the register is to study complications related to the use of CVCs, especially central line associated blood stream infection and mechanical complications (eg, thrombosis, leakage, accidental removal). The database reports on the quality indicators listed in Table 1. submit your manuscript | www.dovepress.com

Dovepress

http://dx.doi.org/10.2147/CLEP.S99508
Clinical Epidemiology 2016:8 46I-464 (Thematic series on clinical quality databases in Denmark) 46 I (c) (i) (5) 2016 Schroder et al. This work is published and licensed by Dove Medical Press Limited. The full terms of this license are available at https://www.dovepress.com/terms. cc. you hereby accept the Terms. Non-commercial uses of the work are permitted without any further permission from Dove Medical Press Limited, provided the work is properly attributed. For

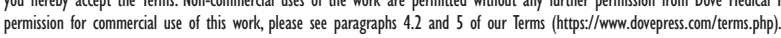


Table I Quality indicators of childhood cancer in Denmark

Number of patients treated according to and reported to an international protocol

5-year relapse-free survival

5-year overall survival

Number of patients dying from complications due to treatment

Days from diagnosis to start of treatment

Number of patients developing a secondary cancer

Number of BSI-related deaths within 30 days after the diagnosis of BSI

Number of BSIs in which the microorganisms are sensitive to the applied

first-line antimicrobial chemotherapy

Number of BSIs per I,000 CVC-days

Number of premature removals of CVC caused by infectious

complications

Abbreviations: BSI, blood stream infection; CVC, central venous catheter.

Finally, registrations of acute toxicity of high-dose methotrexate in children with acute lymphoblastic leukemia and data regarding late effects after treatment of childhood cancer are linked to DCCR.

\section{Study population}

All children below 15 years of age at diagnosis living in Denmark with a diagnosis of a neoplasm according to the International Classification of Diseases 10 (ICD10), including diagnoses DC00-DD48 are included in DCCR.

All tunneled CVCs and all blood stream infections from 2015 and onward are registered in children below 15 years of age at diagnosis (with one of the relevant ICD10 diagnoses).

Since 2008, toxicity data related to high-dose methotrexate infusions in all Danish children and adults with acute lymphoblastic leukemia, treated according to the Nordic NOPHO ALL 2008 protocol, have been registered.

The unique Danish personal identity number allows for unambiguous linkage between DCCR and the Danish National Patient Registry, the National Pathology Registry, and the Danish Cancer Registry (DCR). This linkage allows identification of missing data. Consequently, data regarding the annual cancer incidence rate in children below 15 years of age and their survival status are $100 \%$ complete. In total, $98 \%$ of children with cancer are treated at one of the four regional childhood cancer centers in Denmark (The University Hospitals in Copenhagen, Aarhus, Odense, and Aalborg). Research nurses at the four centers are responsible for retrieving relevant clinical data on all newly diagnosed patients, and for entering these data into the database after validation by a local pediatric oncologist. At the time of diagnosis written informed consent from the parents is obtained for registration of clinical data in DCCR.

\section{Main variables}

The main variables of DCCR are presented in Table 2.

All tumors have been classified according to the Birch and Marsden classification ${ }^{1}$ and the ICD10 classification; and we are in the process of reclassifying patients according to the International Childhood Cancer Classification $3^{2}$ and the ICD-O classifications.

For solid tumors and lymphomas, the date of diagnosis is defined as the date of the first paraclinical investigation (X-ray, ultrasound, computed tomography/magnetic resonance scan) which indicated what later could be pathologically confirmed as a cancer. For leukemia, the date of diagnosis is the day of the bone marrow biopsy/aspiration. The therapy start date is recorded and allows calculation of the diagnostic delay. This is one of the five national quality parameters (see Table 1) by which the regional pediatric oncology centers are evaluated in Denmark.

In each childhood cancer case the applied international treatment protocol is documented within DCCR database. This gives us an opportunity to calculate doses of the individual

Table 2 Main variables in the Danish Childhood Cancer Registry (DCCR)

\section{Cancer data}

CPR

Tumor type

Extent of disease (localization of metastases)

Date of diagnosis

Date of start of therapy

Type of therapy (operation, chemotherapy, irradiation, observation)

Treatment protocol

Reporting of clinical data according to international protocol

Response to initial therapy

Date of cessation of therapy

Relapse (date, localization, treatment and outcome of relapse)

Yearly follow-up

Disease status:

Alive in complete remission

Alive with active disease

Development of secondary malignancy

Death: date and cause of death

Tunneled central venous catheters ( $t$-CVCs)

Type of t-CVC

Date of insertion of $\mathrm{t}-\mathrm{CVC}$

Date of removal of $\mathrm{t}-\mathrm{CVC}$

Cause for removal of $\mathrm{t}-\mathrm{CVC}$

\section{Bacteremia}

Date of bacteremia

Focus for the bacteremia

Microbiological agents

Bacterial drug sensitivity

Antibiotic treatment, type, and length

Result of antibacterial therapy

Abbreviation: CPR, cardiopulmonary resuscitation. 
chemotherapeutic drugs the child has received, which may have implications for the risk of developing late effects of the treatment. All surgeries with the attempt of tumor removal are recorded, including the completeness of tumorectomy. For radiation therapy the total dose and the dates are registered, however data regarding the target or size of the field are not reported. The registered treatment data, which have been agreed upon by the regional pediatric oncology centers in Denmark, are $>95 \%$ complete. Since it is a primary goal that all children should be treated according to the best international protocols and that clinical data should be reported where possible, it is also registered whether patient data have been reported to international studies. In total, $95 \%$ of Danish children with cancer are treated according to an international protocol. Data of $95 \%$ of those patients who are treated according to an open protocol are reported to ongoing international studies. This is another national quality indicator (see Table 1) by which the regional pediatric oncology centers in Denmark are evaluated.

Once a year the registrations in DCCR database are cross-checked with the Danish National Patient Registry to detect missing patients, and with the DCR in order to register secondary cancers which may have developed after the child has survived into adulthood. The lag time for identifying secondary cancers via the DCR is about 1 year. Furthermore, on a yearly basis all patients diagnosed after January 2003 are scrutinized for disease status including relapse and relapse therapy, deaths, and emigration (lost to follow-up) by medical record review and by cross-checking the Danish Civil Registration System.

\section{Main results}

Since 1985 4,944 children below 15 years of age have been registered in DCCR database. There has been no significant change in the incidence of childhood cancer in Denmark since 1985. The annual incidence varies between 15.5 and 22.2 cases per 100,000 children below 15 years of age (Figure 1). The recurrence-free 5 -year survival for all cancers diagnosed between 2003 and 2014 was 74\%, and the 5-year survival has increased significantly and steadily from $72 \%$ in $1985^{3}$ to $86 \%$ in 2014 . Three percent of all newly diagnosed children died from a treatment related cause, mostly children with leukemia and lymphoma. The median number of days from date of diagnosis to initiation of therapy was, in 2014, 7 days and in $80 \%$ of the children it was less than 14 days. Sixty-six secondary cancers have been registered in the population of 4,800 children below 15 years of age diagnosed between 1985 and 2014. Data regarding cancer types and survival are annually transferred electronically to the Nordic databases

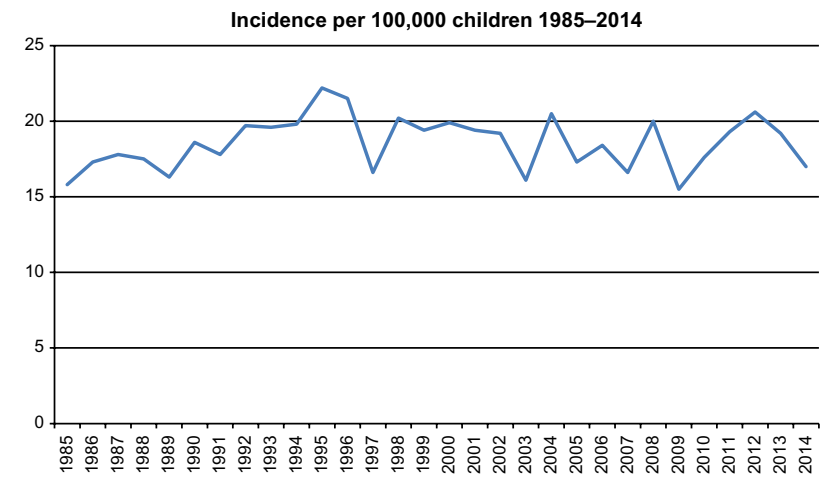

Figure I Yearly cancer incidence in children in Denmark I985-20|4.

for leukemia, lymphoma, and solid tumors in Stockholm, Sweden. ${ }^{4}$ Relevant data are registered to Nordic and other international studies in $95 \%$ of children in which this registration is possible. The entire yearly report of DCCR is published on the homepage of the Danish Pediatric Hematology and Oncology (DAPHO) group. Data from DCCR have been outlined in the annual reports from 2008-2014. ${ }^{5}$

Data from DCCR were important in the $\mathrm{PhD}$ thesis by Ahrensberg, describing the diagnostic delay from the first symptom to start of treatment in children with cancer in Denmark diagnosed between 2008 and 2012. ${ }^{6-8}$

Also, data have been included in a Nordic study of the psycho-social effects of having had cancer during childhood. ${ }^{9-11}$

\section{Funding and administration}

DCCR is funded by the Danish Regions and the annual report is coordinated through the organizational umbrella, the Danish Clinical Registries (RKKP). The Danish Childhood Cancer Foundation provides funding for research nurses at the four centers responsible for data collection and registration.

A DCCR database steering group has been appointed with regional representatives from the pediatric oncology centers at the four university hospitals in Denmark, and from the Department of Clinical Epidemiology, Aarhus University Hospital. The chairman of this group, together with the Department of Clinical Epidemiology will write the annual report which will be discussed in the steering committee before publication.

\section{Conclusion}

The survival of Danish children with cancer since 2003 compares favorably with other international population-based estimates. ${ }^{12,13}$ The annual reports are stimulating for the col- 
laboration within the DAPHO group. The reports focus on areas where there is room for improvement, for example, in connection with the reporting of clinical data according to international clinical trials. Data from DCCR have formed the basis for a series of Danish pediatric oncology $\mathrm{PhD}$ studies. An overall survival rate reaching $86 \%$ in childhood cancer treatment demonstrates a need to further improve the monitoring of patients for the development of late complications of cancer therapy, including secondary cancers. DCCR has been further developed to allow for recording of late effect parameters related to the treatment given.

\section{Acknowledgment}

This paper was funded by: the Program for Clinical Research Infrastructure (PROCRIN) established by the Lundbeck Foundation and the Nordic Foundation and administered by the Danish Regions; The Danish Childhood Cancer Foundation.

\section{Disclosure}

The authors have no conflicts of interest to disclose in this work.

\section{References}

1. Birch JM, Marsden HB. A classification scheme for childhood cancer. Int $J$ Cancer. 1987;40(5):620-624.

2. Steliarova-Foucher E, Stiller C, Lacour B, Kaatsch P. International classification of childhood cancer, third edition. Cancer. 2005;103(7): 1457-1467.
3. de Nully Brown P, Hertz H, Olsen JH, Yssing M, Scheibel E, Jensen OM. Incidence of childhood cancer in Denmark 1943-1984. Int J Epidemiol. 1989;18(3):546-555.

4. nopho.org [homepage on the Internet]. Childhood cancer in the Nordic countries. Report on epidemiologic and therapeutic results from registries and working groups. NOPHO Annual Report 2015. Available from: www.nopho.org/annual report 2015.

5. Schroder H, Wehner PS, Rosthoj S, et al. Annual report Danish childhood cancer registry (DCBR) 2014. Available from: www.dapho.dk/ dbcr-rapporter. Accessed May 15, 2016.

6. Ahrensberg JM, Hansen RP, Olesen F, Schroder H, Vedsted P. Presenting symptoms of children with cancer: a primary-care population-based study. Br J Gen Pract. 2012;62(600):e458-e465.

7. Ahrensberg JM, Schroder H, Hansen RP, Olesen F, Vedsted P. The initial cancer pathway for children - one-fourth wait more than 3 months. Acta Paediatr. 2012;101(6):655-662.

8. Ahrensberg JM, Olesen F, Hansen RP, Schroder H, Vedsted P. Childhood cancer and factors related to prolonged diagnostic intervals: a Danish population-based study. Br J Cancer. 2013;108(6):1280-1287.

9. Johannsdottir IM, Hjermstad MJ, Moum T, et al. Social outcomes in young adult survivors of low incidence childhood cancers. $J$ Cancer Surviv. 2010;4(2):110-118.

10. Johannsdottir IM, Moum T, Hjermstad MJ, et al. Emotional functioning and school contentment in adolescent survivors of acute myeloid leukemia, infratentorial astrocytoma, and Wilms tumor. JAdolesc Young Adult Oncol. 2011;1(3):133-139.

11. Johannsdottir IM, Hjermstad MJ, Moum T, et al. Increased prevalence of chronic fatigue among survivors of childhood cancers: a populationbased study. Pediatr Blood Cancer. 2012;58(3):415-420.

12. Gatta G, Zigon G, Capocaccia R, et al. Survival of European children and young adults with cancer diagnosed 1995-2002. Eur J Cancer. 2009;45(6):992-1005.

13. Gatta G, Botta L, Rossi S, et al. Childhood cancer survival in Europe 1999-2007: results of EUROCARE-5-a population-based study. Lancet Oncol. 2014;15(1):35-47.
Clinical Epidemiology

\section{Publish your work in this journal}

Clinical Epidemiology is an international, peer-reviewed, open access, online journal focusing on disease and drug epidemiology, identification of risk factors and screening procedures to develop optimal preventative initiatives and programs. Specific topics include: diagnosis, prognosis, treatment, screening, prevention, risk factor modification,

Submit your manuscript here: http://www.dovepress.com/clinical-epidemiology-journal

\section{Dovepress}

systematic reviews, risk \& safety of medical interventions, epidemiology \& biostatistical methods, and evaluation of guidelines, translational medicine, health policies \& economic evaluations. The manuscript management system is completely online and includes a very quick and fair peer-review system, which is all easy to use. 